\title{
Persistent low-temperature spin dynamics in the mixed-valence iridate $\mathrm{Ba}_{3} \operatorname{InIr}_{2} \mathrm{O}_{9}$
}

\author{
Tusharkanti Dey, ${ }^{1, *}$ M. Majumder, ${ }^{1}$ J. C. Orain, ${ }^{2}$ A. Senyshyn, ${ }^{3}$ M. Prinz-Zwick,${ }^{4}$ S. Bachus,,${ }^{1}$ Y. Tokiwa,,${ }^{1}$ F. Bert, ${ }^{5}$ \\ P. Khuntia, ${ }^{5}$ N. Büttgen, ${ }^{4}$ A. A. Tsirlin,,${ }^{1, \dagger}$ and P. Gegenwart ${ }^{1, \dagger}$ \\ ${ }^{1}$ Experimental Physics VI, Center for Electronic Correlations and Magnetism, University of Augsburg, 86159 Augsburg, Germany \\ ${ }^{2}$ Laboratory for Muon Spin Spectroscopy, Paul Scherrer Institut, 5232 Villigen PSI, Switzerland \\ ${ }^{3}$ Forschungsneutronenquelle Heinz Maier-Leibnitz, (FRM II), Technische Universität München, 85747 Garching, Germany \\ ${ }^{4}$ Experimental Physics V, Center for Electronic Correlations and Magnetism, University of Augsburg, 86159 Augsburg, Germany \\ ${ }^{5}$ Laboratoire de Physique des Solides, CNRS, Université Paris-Sud, Université Paris-Saclay, 91405 Orsay Cedex, France
}

(Received 28 February 2017; revised manuscript received 10 October 2017; published 8 November 2017)

\begin{abstract}
Using thermodynamic measurements, neutron diffraction, nuclear magnetic resonance, and muon spin relaxation, we establish putative quantum spin-liquid behavior in $\mathrm{Ba}_{3} \operatorname{InIr}_{2} \mathrm{O}_{9}$, where unpaired electrons are localized on mixed-valence $\mathrm{Ir}_{2} \mathrm{O}_{9}$ dimers with $\mathrm{Ir}^{4.5+}$ ions. Despite the antiferromagnetic Curie-Weiss temperature on the order of $10 \mathrm{~K}$, neither long-range magnetic order nor spin freezing are observed down to at least $20 \mathrm{mK}$, such that spins are short-range correlated and dynamic over nearly three decades in temperature. Quadratic power-law behavior of both the spin-lattice relaxation rate and specific heat indicates the gapless nature of the ground state. We envisage that this exotic behavior may be related to an unprecedented combination of the triangular and buckled honeycomb geometries of nearest-neighbor exchange couplings in the mixed-valence setting.
\end{abstract}

DOI: 10.1103/PhysRevB.96.174411

\section{INTRODUCTION}

Frustrated magnets host multiple exotic states, including quantum spin liquids (QSLs). In a QSL, spins are strongly correlated, but quantum fluctuations prevent them from longrange ordering [1,2]. The initial (and subsequently rebutted) proposal of the QSL resonating-valence-bond state on the triangular lattice of Heisenberg spins [3] was followed by similar proposals for several other isotropic (Heisenberg) spin- $\frac{1}{2}$ models, from which the formation of QSLs is now established [4-8]. A few candidate QSL materials proposed over the last decade bear key experimental signatures of this exotic state, including persistent spin dynamics and the absence of long-range order within the experimentally accessible temperature range [9-12].

More recently, QSL states in anisotropic magnets have been explored. Here, the Kitaev model with anisotropic interactions on the honeycomb lattice [13] offers an exact solution for the QSL. Real-world manifestations of the Kitaev physics are found in compounds of $4 d$ and $5 d$ transition metals [14], where large spin-orbit coupling triggers strong intersite magnetic anisotropy. However, none of the Kitaev materials reported to date host the QSL ground state in zero field, and long-range order typically sets in at low temperatures, owing to substantial interactions beyond the Kitaev terms [15,16].

In this paper, we propose an alternative strategy and search for QSL states in the family of mixed-valence $5 d$ oxides, where unpaired electrons are localized on dimers of the Ir atoms. This should facilitate access to hitherto unexplored local electronic states [17] and new regimes of anisotropic exchange interactions. Specifically, we report low-temperature magnetic behavior of the mixed-valence iridate $\mathrm{Ba}_{3} \mathrm{InIr}_{2} \mathrm{O}_{9}$ as a QSL candidate, and confirm its persistent spin dynamics as

\footnotetext{
*tusdey@gmail.com

†altsirlin@gmail.com

philipp.gegenwart@physik.uni-augsburg.de
}

well as the absence of long-range magnetic order down to at least $20 \mathrm{mK}$. We further identify quadraticlike power-law behavior of both specific heat and the spin-lattice relaxation rate and compare these observations to existing theoretical results on QSLs.

$\mathrm{Ba}_{3} \mathrm{InIr}_{2} \mathrm{O}_{9}$ belongs to the family of hexagonal perovskites $A_{3} B M_{2} \mathrm{O}_{9}$. Their structures comprise single $\mathrm{BO}_{6}$ octahedra and $M_{2} \mathrm{O}_{9}$ dimers of two face-sharing $M \mathrm{O}_{6}$ octahedra [Fig. 1(a)]. When a magnetic ion occupies the $B$ site, triangular interaction geometry is formed, as in $\mathrm{Ba}_{3} \mathrm{CoSb}_{2} \mathrm{O}_{9}$, which is arguably the best model spin- $\frac{1}{2}$ antiferromagnet on the triangular lattice [18-20]. Placing a $4 d$ or $5 d$ ion into the $B$ site or into one of the $M$ sites could give rise to a triangular system with leading Kitaev interactions [21,22], but experimental implementation of this idea is hindered by the strong $B / M$ site mixing that occurs, e.g., in $\mathrm{Ba}_{3} \mathrm{IrTi}_{2} \mathrm{O}_{9}$ [23-25]. Alternatively, Ir could be introduced into both $M$ sites while keeping the $B$ site nonmagnetic, but for integer valence of $M$ such a spin dimer would simply condense into a nonmagnetic singlet [26,27]. Mixed-valence systems with both $M$ sites occupied by a magnetic $5 d$ ion are possible too [28-31] and seem to be more promising for finding a QSL because unpaired electrons localized on the dimers appear. Note that such mixed-valence dimers with an unpaired electron delocalized between the two $\mathrm{Ir}^{4.5+}$ ions are very different from more conventional spin dimers formed by two magnetic ions holding one unpaired electron each.

Guided by this idea, we synthesized polycrystalline samples of $\mathrm{Ba}_{3} \mathrm{InIr}_{2} \mathrm{O}_{9}$. From our detailed study using neutron diffraction, magnetization and specific heat measurements, muon spin relaxation $(\mu \mathrm{SR})$, and nuclear magnetic resonance (NMR) we establish a gapless and, potentially, spin-liquid ground state in $\mathrm{Ba}_{3} \mathrm{InIr}_{2} \mathrm{O}_{9}$.

\section{EXPERIMENTAL DETAILS}

Polycrystalline samples of $\mathrm{Ba}_{3} \mathrm{InIr}_{2} \mathrm{O}_{9}$ were prepared by a conventional solid-state reaction method [29]. Stoichiometric 

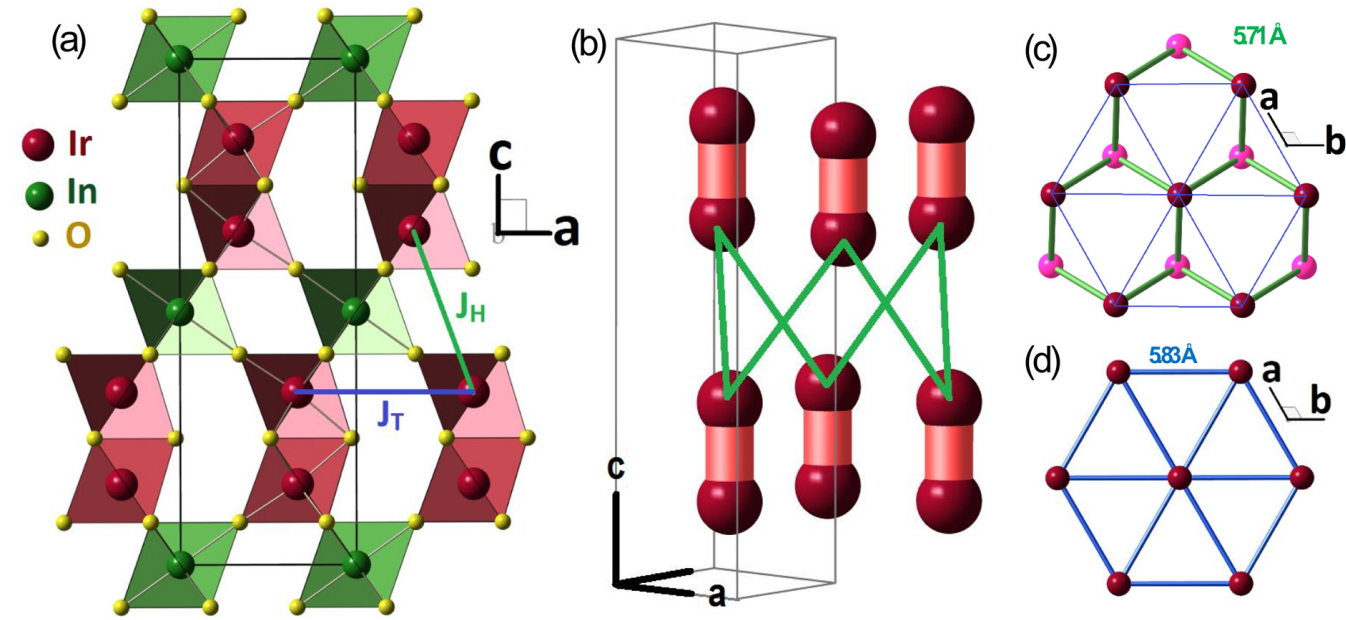

FIG. 1. (a) The crystal structure of $\mathrm{Ba}_{3} \mathrm{InIr}_{2} \mathrm{O}_{9}$ comprising the $\mathrm{IrO}_{6}$ (red) and $\mathrm{InO}_{6}$ (green) octahedra. The $\mathrm{Ba}$ atoms are omitted for clarity. (b) Magnetic moments are localized within the Ir dimers (shown as dumbbells). The interplane couplings $J_{H}$ are shown as green lines. (c) The interplane coupling $J_{H}$ forming the buckled honeycomb spin lattice. (d) The intraplane coupling $J_{T}$ forming the triangular spin lattice.

amounts of high-purity $\mathrm{BaCO}_{3}, \mathrm{In}_{2} \mathrm{O}_{3}$, and $\mathrm{Ir}$ metal powder were mixed thoroughly, pressed into pellets, and calcined at $900{ }^{\circ} \mathrm{C}$ for $12 \mathrm{~h}$. Further, the pellet was crushed into powder, mixed well, pelletized, and fired at $1300{ }^{\circ} \mathrm{C}$ for 4 days with several intermediate grindings. Neutron diffraction data were collected at the high-resolution instrument SPODI [32] at Forschungsneutronenquelle Heinz Maier-Leibnitz (FRM II, Technische Universität München) using a wavelength of $1.55 \AA$. JANA2006 software [33] was used for structure refinement.

Magnetization measurements were carried out in a Quantum Design 5-T superconducting quantum interference device (SQUID) magnetometer and in a Quantum Design 14-T physical property measurement system (PPMS) equipped with the vibrating sample magnetometer in the temperature range 2-350 K. Additional high-temperature data extending up to $650 \mathrm{~K}$ were collected in the SQUID magnetometer using a powder sample enclosed in a thin-walled quartz tube.

Heat-capacity measurements in the temperature range 0.4-200 K were performed in a Quantum Design PPMS using the ${ }^{3} \mathrm{He}$ insert. Low-temperature measurements in the 0.08$1 \mathrm{~K}$ range were performed using a quasiadiabatic heat-pulse method, adapted to a dilution refrigerator.

$\mu$ SR experiments were done on two different spectrometers at the Paul Scherrer Institute (Switzerland), LTF for temperatures from $20 \mathrm{mK}$ up to $750 \mathrm{mK}$ and Dolly for temperatures from $250 \mathrm{mK}$ up to $200 \mathrm{~K}$. For the Dolly experiment, about $300 \mathrm{mg}$ of the polycrystalline sample was mounted on a thin copper plate inside the ${ }^{3} \mathrm{He}$ cryostat. In order to ensure good thermal contact, we glued the sample with GE varnish. We used the veto mode, which allowed us to get rid of the background signal from the sample holder. Therefore, the acquired signal is due to muons that stopped inside the sample. For the LTF experiment we used the same sample, again glued with GE on a silver plate. By comparing the results obtained between 250 and $750 \mathrm{mK}$ on both the spectrometers, we were able to get rid of the experimental background on LTF.

${ }^{115}$ In nuclear magnetic resonance (NMR) experiments were carried out with our home-built spectrometer with the dilution-fridge insert. The measurements were performed in the field-sweep mode at a fixed frequency of $70 \mathrm{MHz}$ down to $24 \mathrm{mK}$. The spin-echo intensity was obtained by integrating over the spin echo in the time domain. The final spectrum was constructed by plotting the spin-echo intensity as a function of the applied field.

\section{RESULTS}

\section{A. Crystal structure}

Rietveld refinement of room-temperature neutron diffraction data (Fig. 2) confirms hexagonal crystal structure $\left(\mathrm{Pb}_{3} / \mathrm{mmc}\right)$. However, at $3.4 \mathrm{~K}$ peak splitting of the 203 and 204 reflections, as well as a visible broadening of other peaks, indicated that the symmetry is reduced to monoclinic. The $3.4 \mathrm{~K}$ data were refined in the $C 2 / c$ space group, similar to

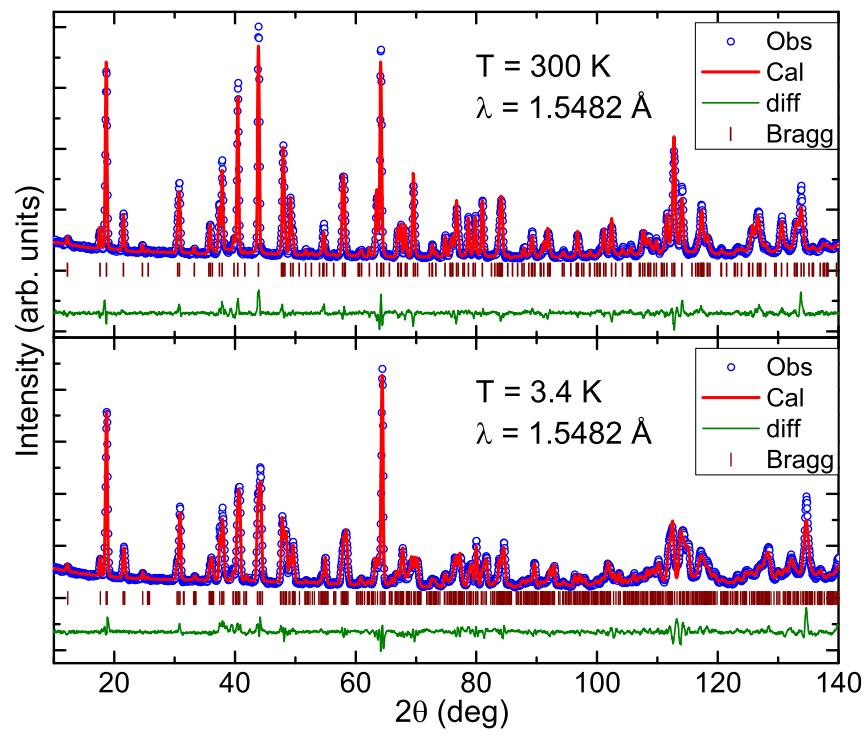

FIG. 2. Rietveld refinement of neutron diffraction data at room temperature (top) and at $3.4 \mathrm{~K}$ (bottom). 
TABLE I. Refined atomic positions for $\mathrm{Ba}_{3} \operatorname{InIr}_{2} \mathrm{O}_{9}$ at $300 \mathrm{~K}$. $U_{\text {iso }}$ are isotropic atomic displacement parameters (in $10^{-2} \AA^{2}$ ). Lattice parameters: $a=5.8316(1) \AA, c=14.4877(4) \AA, P 6_{3} / m m c, R_{I}=$ $0.039, R_{p}=0.051$.

\begin{tabular}{lccccc}
\hline \hline & & $x / a$ & $y / b$ & $z / c$ & $U_{\text {iso }}$ \\
\hline $\mathrm{Ba} 1$ & $2 b$ & 0 & 0 & 0.25 & $1.3(1)$ \\
$\mathrm{Ba} 2$ & $4 f$ & $\frac{1}{3}$ & $\frac{2}{3}$ & $0.9103(2)$ & $0.9(1)$ \\
$\mathrm{In}$ & $2 a$ & 0 & 0 & 0 & $0.4(1)$ \\
$\mathrm{Ir}$ & $4 f$ & $\frac{1}{3}$ & $\frac{2}{3}$ & $0.1590(1)$ & $1.29(3)$ \\
$\mathrm{O} 1$ & $6 h$ & $0.4867(2)$ & $0.5133(2)$ & 0.25 & $1.40(5)$ \\
$\mathrm{O} 2$ & $12 k$ & $0.1715(2)$ & $0.3430(4)$ & $0.0841(1)$ & $2.1(1)$ \\
\hline \hline
\end{tabular}

other hexagonal perovskites [28,29,34]. Both hexagonal and monoclinic structures feature a single crystallographic position of $\operatorname{Ir}$ (Tables I and II), suggesting the true intermediate-valence $\mathrm{Ir}^{4.5+}$ state. This is different from, e.g., $\mathrm{Ba}_{5} \mathrm{AlIr}_{2} \mathrm{O}_{11}$, where two sites of the dimer belong to two different crystallographic positions, thus making possible charge redistribution within the dimer [35].

In contrast to $\mathrm{Ba}_{3} \mathrm{IrTi}_{2} \mathrm{O}_{9}$ with its $35 \%-40 \%$ site mixing [23,25], our mixed-valence $\mathrm{Ba}_{3} \mathrm{InIr}_{2} \mathrm{O}_{9}$ shows a high degree of structural order. We were able to obtain reasonable atomic displacement parameters in the fully ordered models of both hexagonal and monoclinic structures (Tables I and II). On the other hand, deliberate admixing of In into the Ir position and vice versa leads to a marginal reduction in the refinement residuals and 2.8(5)\% site mixing [36]. We believe that the diffraction data alone may not distinguish between the fully ordered structure and the weak-site-mixing scenario. Further studies, such as direct imaging with high-resolution electron microscopy, would be useful to resolve this issue.

The low-temperature monoclinic distortion is primarily related to the tilting of the $\mathrm{IrO}_{6}$ and $\mathrm{InO}_{6}$ octahedra. It has nearly no effect on relative positions of the Ir atoms. For example, the Ir-Ir distance within the dimer shrinks from $2.637(2) \AA$ at $300 \mathrm{~K}$ to 2.599 (4) $\AA$ at $3.4 \mathrm{~K}$, presumably due to thermal expansion. The Ir-Ir distances between the dimers do not change at all: compare 5.832(1) $\AA$ at $300 \mathrm{~K}$ to $5.813(8)$ and 5.815(4) $\AA$ at $3.4 \mathrm{~K}$ for the nearest-neighbor Ir-Ir distances in the $a b$ plane. Given this negligible structural effect and the

TABLE II. Refined atomic positions for $\mathrm{Ba}_{3} \operatorname{InIr}_{2} \mathrm{O}_{9}$ at $3.4 \mathrm{~K}$. $U_{\text {iso }}$ are isotropic atomic displacement parameters (in $10^{-2} \AA^{2}$ ). Lattice parameters: $a=5.8152(3) \AA, b=10.0680(5) \AA, c=14.4619(6) \AA$, $\beta=90.854(3)^{\circ}, C 2 / c, R_{I}=0.039, R_{p}=0.061$. Atomic displacements parameters for oxygen were constrained.

\begin{tabular}{lccccc}
\hline \hline & \multicolumn{1}{c}{$y / a$} & \multicolumn{1}{c}{$z / c$} & $U_{\text {iso }}$ \\
\hline $\mathrm{Ba} 1$ & $4 e$ & 0 & $0.0010(15)$ & 0.25 & $1.0(1)$ \\
$\mathrm{Ba} 2$ & $8 f$ & $0.0049(9)$ & $0.3360(11)$ & $0.0889(3)$ & $0.1(1)$ \\
$\mathrm{In}$ & $4 a$ & 0 & 0 & 0 & $0.2(1)$ \\
$\mathrm{Ir}$ & $8 f$ & $-0.0081(5)$ & $0.3334(6)$ & $0.8397(2)$ & $1.0(1)$ \\
$\mathrm{O} 1$ & $4 e$ & 0 & $0.4918(10)$ & 0.75 & $1.2(1)$ \\
$\mathrm{O} 2$ & $8 f$ & $0.230(2)$ & $0.2573(8)$ & $0.7541(4)$ & $1.2(1)$ \\
$\mathrm{O} 3$ & $8 f$ & $-0.018(2)$ & $0.1717(8)$ & $0.9140(6)$ & $1.2(1)$ \\
$\mathrm{O} 4$ & $8 f$ & $0.226(1)$ & $0.4158(9)$ & $0.9251(4)$ & $1.2(1)$ \\
$\mathrm{O} 5$ & $8 f$ & $-0.263(1)$ & $0.4144(9)$ & $0.9079(5)$ & $1.2(1)$ \\
\hline \hline
\end{tabular}

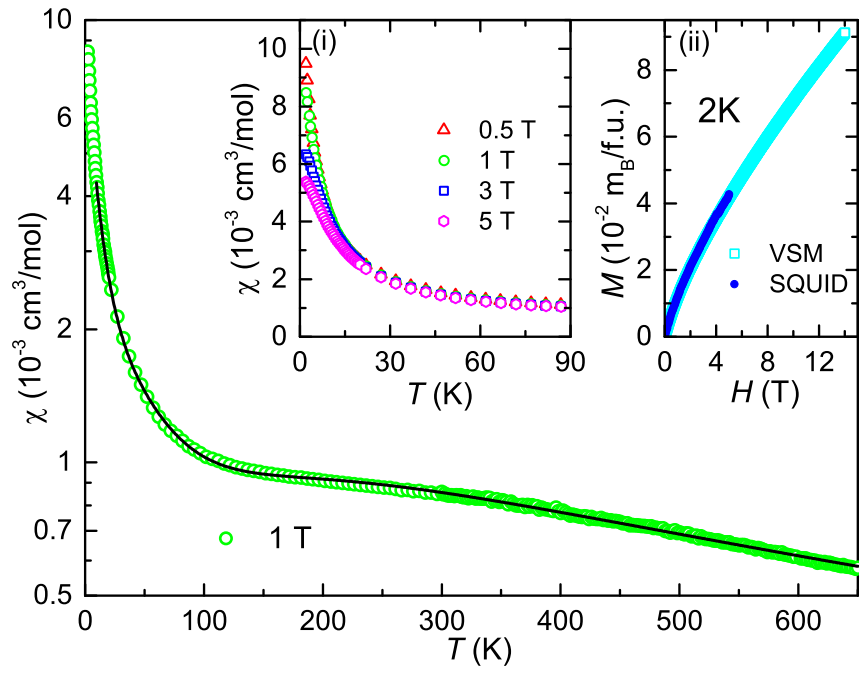

FIG. 3. Magnetic susceptibility of $\mathrm{Ba}_{3} \operatorname{InIr}_{2} \mathrm{O}_{9}$ at $1 \mathrm{~T}$ is shown in semilog scale in the temperature range $2-650 \mathrm{~K}$. The black solid line is the fit of the data with Eq. (1). Insets: (i) Magnetic susceptibility of $\mathrm{Ba}_{3} \mathrm{InIr}_{2} \mathrm{O}_{9}$ shown as a function of temperature at various applied fields. (ii) Isothermal magnetization curve measured at $2 \mathrm{~K}$ by SQUID (up to $5 \mathrm{~T}$ ) and a vibrating-sample magnetometer (VSM; up to $14 \mathrm{~T}$ ).

absence of any signatures in thermodynamic measurements, we conclude that the hexagonal-to-monoclinic phase transition should be an effect of structural (geometrical) origin and bears no relation to the magnetism of $\mathrm{Ba}_{3} \mathrm{InIr}_{2} \mathrm{O}_{9}$. Determination of the exact transition temperature requires further dedicated diffraction experiments at intermediate temperatures and lies beyond the scope of our present study.

\section{B. Magnetic susceptibility}

The temperature dependence of the magnetic susceptibility $(\chi=M / H)$ measured in various applied fields is shown in inset (i) of Fig. 3. Down to $2 \mathrm{~K}$, we did not observe any anomaly or divergence of field-cooled and zero-field-cooled data, suggesting the absence of long-range ordering and spin freezing.

The susceptibility follows the Curie-Weiss (CW) behavior between 10 and $70 \mathrm{~K}$. At higher temperatures (see Fig. 3), the data deviate from the $\mathrm{CW}$ law due to thermal changes in the mixed-valence $\mathrm{Ir}_{2} \mathrm{O}_{9}$ dimers that can adopt different electronic configurations [17]. A tentative van Vleck fit with the three-level model

$$
\begin{aligned}
\chi(T)= & \chi_{0}+\frac{N_{A} \mu_{B}^{2}}{3 k_{B}(T-\theta)} \\
& \times \frac{3}{4} \frac{g_{0}^{2}+5 g_{1}^{2} e^{-\Delta_{1} / k_{B} T}+5 g_{2}^{2} e^{-\Delta_{2} / k_{B} T}}{1+e^{-\Delta_{1} / k_{B} T}+e^{-\Delta_{2} / k_{B} T}},
\end{aligned}
$$

where $N_{A}$ is Avogadro's number, $g_{0}$ is the electronic $g$-factor for the ground state, and $g_{1}$ and $g_{2}$ are $g$-factor for the excited states, which are separated from the ground state by energy gaps of $\Delta_{1}$ and $\Delta_{2}$, respectively, yields a decent description of the magnetic susceptibility up to at least $650 \mathrm{~K}$. This fitting function can be understood as follows. In the absence of electronic correlations and spin-orbit coupling, nine electrons occupying six $t_{2 g}$ orbitals of two Ir atoms give rise to $S=\frac{1}{2}$ 
and $S=\frac{3}{2}$ states depending on the filling of the molecular orbitals of the dimer [37]. The effect of spin-orbit coupling is taken into account by introducing electronic $g$ factors as fitting parameters. Additionally, we assumed that the $S=\frac{3}{2}$ state splits into two because fitting with one excited state was not successful, whereas two distinct excited states provide a good description of the susceptibility in the broad temperature range.

Fitting the susceptibility with Eq. (1) yields $\chi_{0}=-6.8 \times$ $10^{-5} \mathrm{~cm}^{3} / \mathrm{mol}, \theta=-6.8 \mathrm{~K}, \Delta_{1}=107 \mathrm{~K}, \Delta_{2}=472 \mathrm{~K}$, $g_{0}=0.872, g_{1}=0.678$, and $g_{2}=1.810$. The relatively low value of $\Delta_{1}$ explains the deviation from the Curie-Weiss behavior already above $70 \mathrm{~K}$. The obtained $\Delta_{1}$ and $\Delta_{2}$ are of the same order of magnitude as in the mixed-valence ruthenates isostructural to $\mathrm{Ba}_{3} \mathrm{InIr}_{2} \mathrm{O}_{9}$ [37]. The paramagnetic effective moments are $\mu_{\text {eff }}=0.76 \mu_{B}$ in the ground state, $\mu_{\text {eff }, 1}=1.31 \mu_{B}$ in the first excited state, and $\mu_{\text {eff }, 2}=3.51 \mu_{B}$ in the second excited state.

For the rest of this paper, we focus on the low-temperature regime below $70 \mathrm{~K}$ with a paramagnetic effective moment of $\mu_{\text {eff }}=0.76 \mu_{B} /$ dimer, which is comparable to the values reported for mixed-valence iridates earlier [35,38]. Antiferromagnetic couplings between magnetic moments localized on the mixed-valent dimers are confirmed by the negative CurieWeiss temperature $\theta_{\chi}=-7 \mathrm{~K}$. The isothermal magnetization curve at $2 \mathrm{~K}$ [see inset (ii) of Fig. 3] does not show any sign of saturation up to $14 \mathrm{~T}$. This weak sensitivity to the field may be due to the strongly reduced $g$-factor (only $44 \%$ of its spin-only value) that lessens the effect of the external field.

\section{Specific heat}

The first insight into the low-temperature magnetism is obtained from the specific-heat data. A broad peak in $C_{p} / T$ is observed around $1.6 \mathrm{~K}$ [Fig. 4(a)], indicative of a crossover between the paramagnetic (thermally disordered) and spinliquid (quantum disordered) regimes [39,40], which is further confirmed by the increase in the muon zero-field relaxation rate around the same temperature (Sec. IIID). The peak shifts toward lower temperatures in the applied field, although the changes are relatively small even at $14 \mathrm{~T}$, and no transition anomaly is observed.

Heat capacity was further measured at temperatures well below the broad maximum. For an insulating material, total heat capacity is a sum of the magnetic, lattice, and nuclear contributions, $C_{p}=C_{m}+C_{\text {lat }}+C_{\text {nuc }}$. To extract the magnetic specific heat of the material, we need to subtract the lattice part and nuclear part from the total specific heat $C_{p}$. In the absence of a suitable nonmagnetic analog, we fitted the measured specific heat with $C_{p}=\beta T^{3}$ in the range 14-20 K [41]. The fitting of the lattice part yields $\beta=1.45 \mathrm{~mJ} \mathrm{~mol}^{-1} \mathrm{~K}^{-4}$ and the Debye temperature $\Theta_{D}=272 \mathrm{~K}$. The fitted curve is extrapolated to low temperatures and taken as the lattice part $C_{\text {lat }}$ that was subtracted from the experimental data.

At low temperatures, the nuclear contribution becomes prominent. To extract the magnetic heat capacity, we adopted the following procedure:

(1) We fitted the $C_{p}$ data for each field $B$ from lowest $T$ up to 300-400 mK with $C_{p}=\alpha / T^{2}+C_{0} T^{\gamma}$ [41], where $\alpha / T^{2}$ stands for $C_{\text {nuc }}$ and power-law behavior of $C_{\text {mag }}$ is assumed.
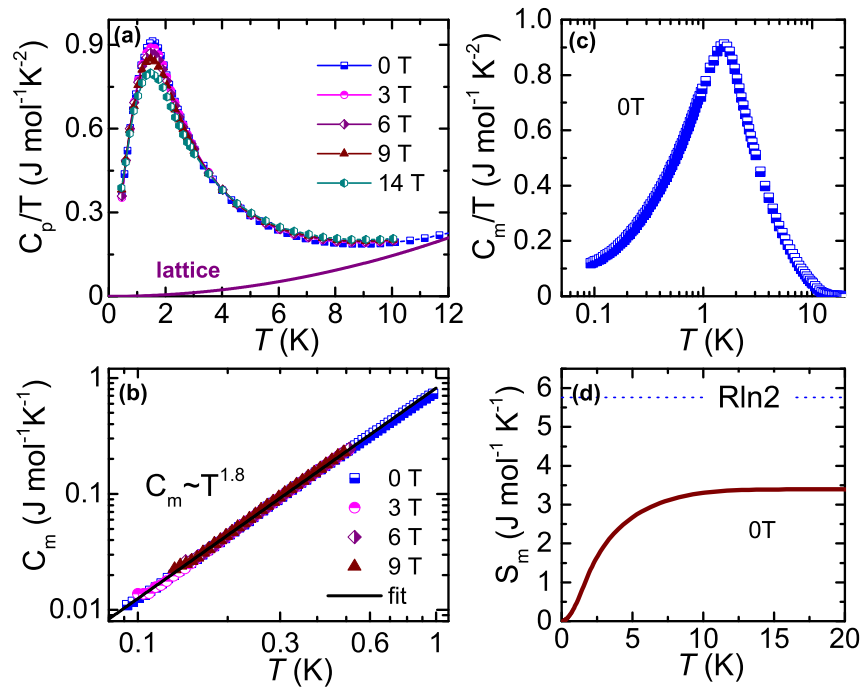

FIG. 4. (a) Temperature dependence of $C_{p} / T$ at various fields together with the lattice contribution. (b) The low-temperature magnetic heat capacity at different fields, shown in log-log scale. The solid line indicates the power law. (c) The temperature dependence of the zero-field magnetic heat capacity divided by temperature $\left(C_{m} / T\right)$, shown in semilog scale. (d) The entropy change $S_{m}$ at zero field.

(2) For each field, the magnetic heat capacity $C_{m}$ is obtained as $C_{m}=C_{p}-C_{\text {nuc }}$, whereas $C_{\text {lat }}$ is negligible in this temperature range.

(3) Reliability of the $\alpha$ values is verified by plotting the field dependence $\alpha\left(B^{2}\right)$, which was linear, as expected [41].

After subtracting the nuclear contribution [41], we arrive at the robust power-law behavior $C_{m}=C_{0} T^{\gamma}$, with $C_{0}=$ $832 \mathrm{~mJ} \mathrm{~mol}^{-1} \mathrm{~K}^{-2.83}$ and $\gamma=1.83$ [Fig. 4(b)]. This behavior gives the first indication of a gapless ground state because, otherwise, low-energy excitations over a spin gap would give rise to the exponential decay of $C_{m}$ at low temperatures. The power-law behavior persists up to at least $14 \mathrm{~T}$, but above $9 \mathrm{~T}$ the exponent $\gamma$ is slightly reduced [41].

By integrating $C_{m} / T$ in zero field [see Fig. 4(c)] within the temperature range from 0.08 to $20 \mathrm{~K}$, we estimated a magnetic entropy change of $3.4 \mathrm{~J} \mathrm{~mol}^{-1} \mathrm{~K}^{-1}$ [see Fig. 4(d)], which is about $60 \%$ of the entropy expected for spin- $\frac{1}{2}$. Part of the magnetic entropy should then be released at higher temperatures, where the magnetic contribution is concealed behind a much larger lattice term. We note here that a twostep release of the magnetic entropy (and, consequently, two well-separated peaks of the magnetic specific heat $C_{m}$ ) is not uncommon in frustrated magnets $[42,43]$.

\section{D. $\mu \mathrm{SR}$}

Whereas thermodynamic measurements provide the first hints towards the absence of long-range magnetic order in $\mathrm{Ba}_{3} \mathrm{InIr}_{2} \mathrm{O}_{9}$, experimental evidence for the QSL formation is not complete without a local probe. To this end, we use $\mu \mathrm{SR}$, which is a very sensitive technique to detect static local fields arising from weak long-range order or spin freezing.

The relaxation curves of the muon polarization in zero field (ZF) are shown in Fig. 5. The absence of oscillations in the $\mathrm{ZF}$ signal and the lack of the polarization recovery to $1 / 3$ 


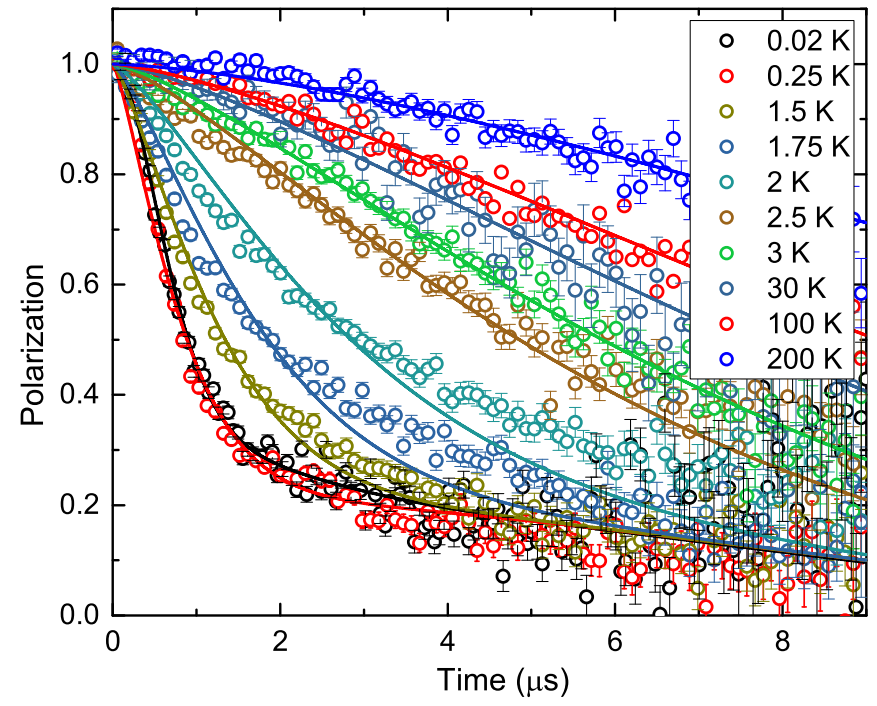

FIG. 5. Muon polarization curves at various temperatures measured under zero field (ZF) along with their fit with Eq. (2).

indicate the absence of any frozen moments in $\mathrm{Ba}_{3} \mathrm{InIr}_{2} \mathrm{O}_{9}$ in the temperature range from $200 \mathrm{~K}$ down to $20 \mathrm{mK}$. The polarization curves can be fitted with the following equation, which is a combination of the depolarization due to the muon coupled to the In nuclear magnetism (Kubo-Toyabe Gaussian function) and depolarization due to the electronic magnetism evolving with temperature:

$P(t)=f e^{-\left(\lambda_{\mathrm{ZF}} t\right)^{\beta}}+(1-f)\left\{\frac{1}{3}+\frac{2}{3}\left[1-(\sigma t)^{2}\right] e^{-\frac{(\sigma t)^{2}}{2}}\right\}$.

Here, $f=0.786(2)$ is the fraction of muons coupled to the electronic magnetism, $\sigma=0.094(2) \mu \mathrm{s}^{-1}$ is the nuclear depolarization rate, $\beta=1.308$ (7) is the stretched exponent, and $\lambda$ is the electronic depolarization rate. The stretched exponent $\beta$ is independent of field and temperature. The small deviation from unity could be due to a small distribution of the muon sites close to the In ions.

The ZF $\mu^{+}$relaxation rate $\lambda_{\mathrm{ZF}}$ obtained from fitting the $\mathrm{ZF}$ muon depolarization curves is shown as a function of temperature in Fig. 6(b). At high temperatures (20 to $3 \mathrm{~K}$ ), $\lambda_{\text {ZF }}$ remains constant at $\sim 0.12 \mu \mathrm{s}^{-1}$, which is consistent with the paramagnetic fluctuations of Ir moments according to the Bloembergen-Purcell-Pound theory [44].

From $3 \mathrm{~K}$ down to $1 \mathrm{~K}, \lambda_{\mathrm{ZF}}$ increases with decreasing temperature. The enhancement of $\lambda_{\mathrm{ZF}}$ in a narrow temperature window indicates a slowing down of Ir spin fluctuations due to the development of strong short-range correlations, a common feature seen in other QSL candidates $[10,11,40]$. Upon further cooling, $\lambda_{\mathrm{ZF}}$ shows temperature-independent plateau-like behavior between $1 \mathrm{~K}$ and $20 \mathrm{mK}$. The plateau-like behavior in $\lambda_{\mathrm{ZF}}$ vs $T$ [see Fig. 6(b)] has also been observed in several other QSL compounds.

To verify if the plateau-like behavior originates from the muons directly coupled to the frustrated spins [45] or the muons coupled to defects [46,47], we have performed $\mu \mathrm{SR}$ experiments under a transverse field of $0.4 \mathrm{~T}$ and estimated the $\mu$ SR line shift $K^{\mu}$ as a function of temperature [48], as shown in Fig. 7(a). $K^{\mu}$ increases with decreasing temperature and saturates below $3 \mathrm{~K}$, from which point $\lambda$ [see Fig. 6(b)]

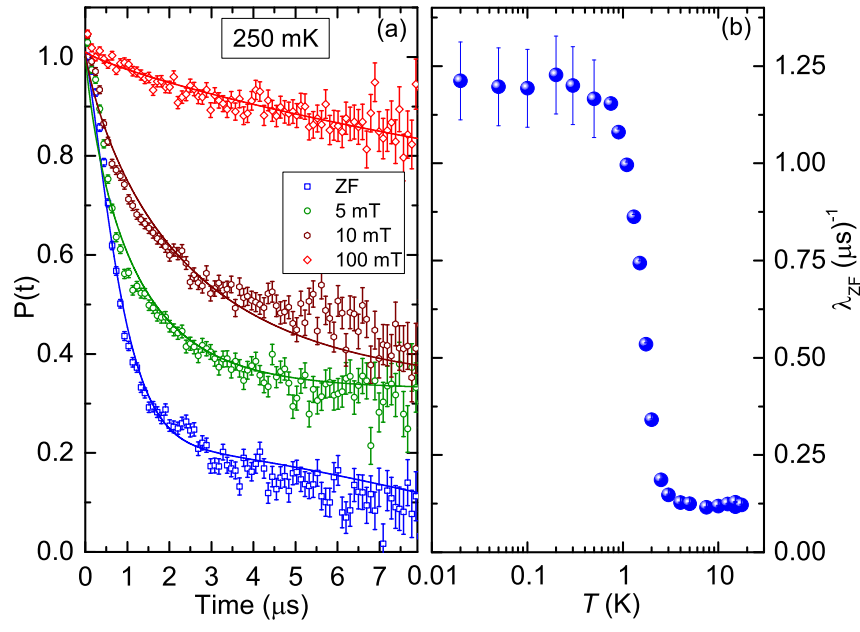

FIG. 6. (a) Muon polarization curves at $250 \mathrm{mK}$ with various applied magnetic fields, shown along with their fitting (see text). (b) Temperature dependence of $\lambda_{\mathrm{ZF}}$ at zero applied magnetic field.

starts increasing. This indicates that antiferromagnetic (AFM) spin fluctuations dominate at low temperature in $\mathrm{Ba}_{3} \operatorname{InIr}_{2} \mathrm{O}_{9}$. The similarity between the temperature dependences of $K^{\mu}$ and the NMR shift $K_{\mathrm{NMR}}$ [see Fig. 7(a)] proves that the muons are directly coupled to the Ir moments.

In order to identify if the origin of the depolarization rate is static or dynamic, we performed decoupling experiments

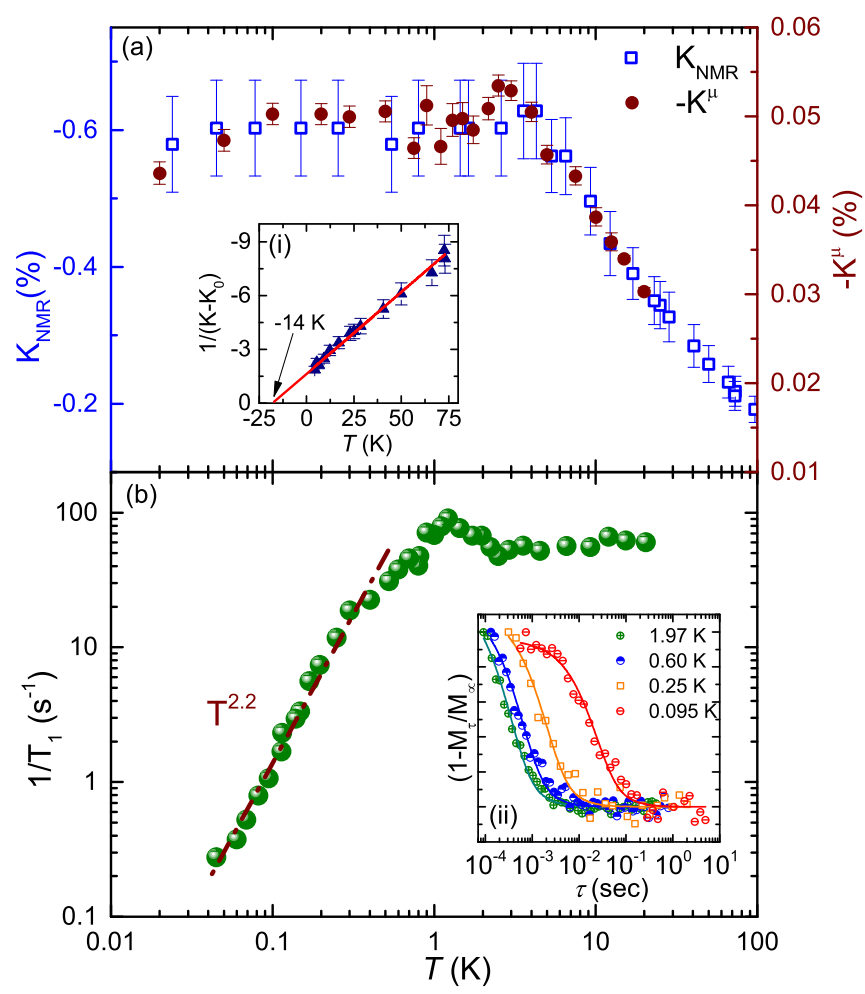

FIG. 7. (a) Temperature dependence of the NMR $\left(K_{\mathrm{NMR}}\right.$; left axis) and $\mu \mathrm{SR}\left(-K^{\mu}\right.$; right axis) line shifts. (b) Temperature dependence of $1 / T_{1}$. The dashed line is a guide to the eye for the power-law $\propto T^{2.2}$ behavior. Insets: (i) $1 /\left(K-K_{0}\right)$ as a function of temperature with the linear $\mathrm{CW}$ fit. (ii) The nuclear magnetization recovery curves along with their fitting at different temperatures. 
at $250 \mathrm{mK}$ [shown in Fig. 6(a)] applying longitudinal fields from 5 up to $100 \mathrm{mT}$. If the low-temperature plateau in $\lambda_{\mathrm{ZF}}$ arises from a static field, the size of this field can be estimated as $B_{\text {loc }}=\lambda / \gamma_{\mu} \approx 1.4 \mathrm{mT}$, where $\gamma_{\mu}=135.5 \times 2 \pi \mathrm{s}^{-1} \mu \mathrm{T}^{-1}$ is the gyromagnetic ratio for muons. The static field can then be decoupled by applying an external magnetic field $\simeq 14 \mathrm{mT}$, which is one order of magnitude higher than $B_{\text {loc }}$. The polarization curve under a field of $100 \mathrm{mT}$ does not show the signs of full polarization, suggesting that the plateau does not originate from a static internal field and the spins are dynamic in nature even at the lowest temperature of $20 \mathrm{mK}$, as expected in a QSL. The plateau-like behavior of $\lambda_{\mathrm{ZF}}$ toward the lowest temperature indicates much slower spin dynamics of the material compared to the $\mu$ SR time window. It is another characteristics of QSL candidates [10,11,40].

\section{E. NMR}

Nuclear magnetic resonance is a concurrent probe for local magnetic fields and spin dynamics. ${ }^{115}$ In NMR spectra measured at $70 \mathrm{MHz}$ in the temperature range $0.024-138 \mathrm{~K}$ are shown in Fig. 8(a). At high temperatures (for example, at $138 \mathrm{~K}$ ), the spectra exhibit one single isotropic line which indicates the unique crystallographic site for indium, consistent with our structural analysis. The spectra can be described satisfactorily assuming the $I=9 / 2$ nuclei with the $380-\mathrm{kHz}$ quadrupolar coupling constant $v_{Q}$.

Below about $100 \mathrm{~K}$, we observed a second line, which remained unshifted within the entire temperature range. This second line presumably originates from trace amounts $(0.9 \%)$ of a nonmagnetic impurity phase present in the sample. To estimate the impurity contribution in the spectral line shape, we measured the spectra at several temperatures with $\tau=50 \mu$ s and $\tau=200 \mu$ s, where $\tau$ is the pulse separation in the spin-echo sequence $\pi / 2-\tau-\pi$. The intrinsic line has a much shorter spin-lattice relaxation time $T_{1}$ as well as spin-spin relaxation time $T_{2}$ compared to the impurity line [41]. Hence, it is expected that with longer $\tau$ the intrinsic part of the spectra is already relaxed, whereas the impurity contribution remains. A comparison of the spectra corresponding to different $\tau$ measured at $T=25 \mathrm{~K}$ is shown in Fig. 8(c).

The spectra are described at all temperatures below $100 \mathrm{~K}$ with two Gaussian lines, one for the magnetic (intrinsic) contribution and the other one for a nonmagnetic (impurity) contribution, as shown in Fig. 8(a). At low temperatures, the two lines (the intrinsic line and the impurity line) merge together. To estimate the Knight shift of the intrinsic line, we have used the peak of the intrinsic contribution marked with the green arrows in Fig. 8(a). This peak shifts towards higher fields with decreasing temperature, yielding the local spin susceptibility $K$. In general,

$$
K=K_{0}+\left(A_{\mathrm{hf}} / N_{A} \mu_{B}\right) \chi(T),
$$

where $K_{0}$ is the temperature-independent part of the line shift $K$ and $N_{A}$ is Avogadro's number. The hyperfine coupling constant is estimated at $A_{\mathrm{hf}}=-1.675 \mathrm{~T} / \mu_{B}$ [41]. The temperature dependence of the ${ }^{115}$ In line shift $K$ is shown in Fig. 7(a). A continuous increase in the line shift $K(T)$ with decreasing temperature from 100 down to $\sim 4 \mathrm{~K}$ indicates the development of spin correlations. The inverse of the

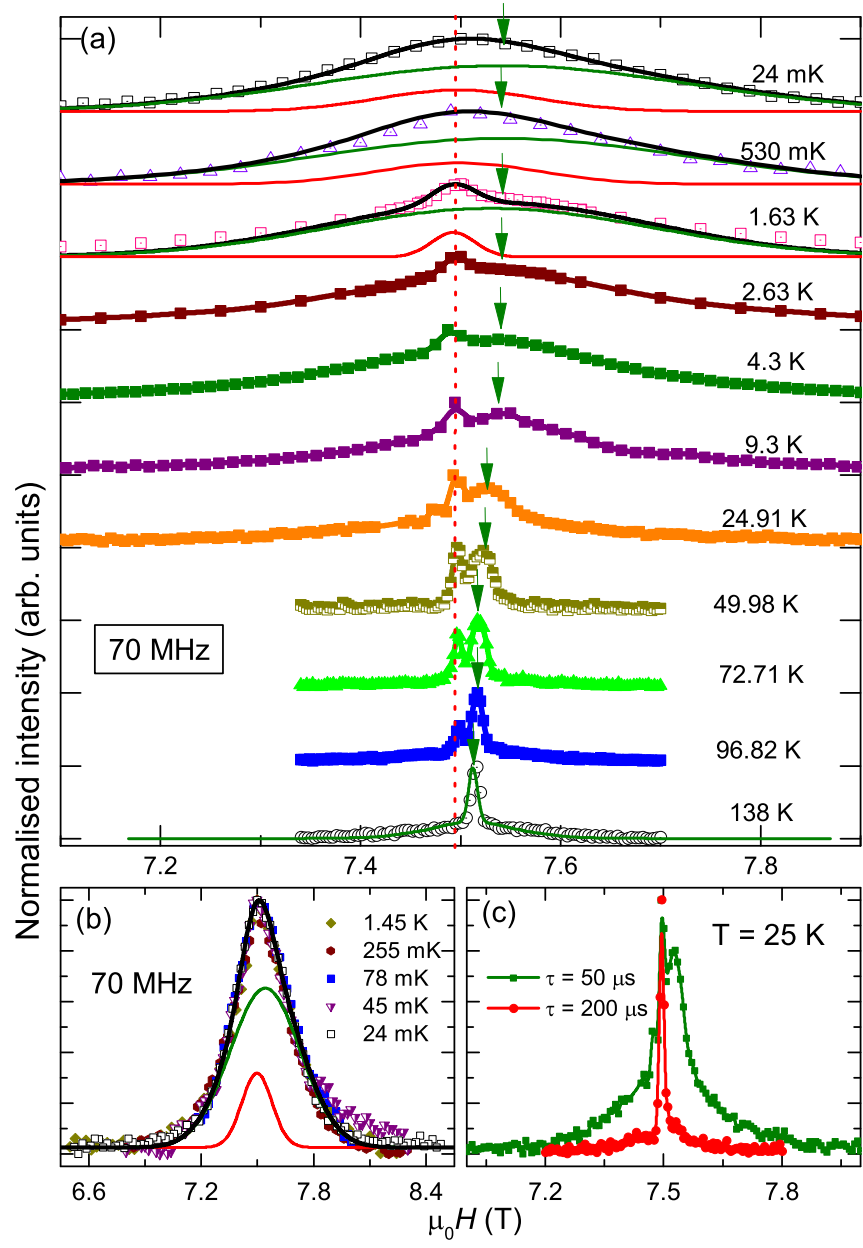

FIG. 8. (a) Temperature evolution of NMR spectra measured at $70 \mathrm{MHz}$. Individual spectra are shifted along the $y$ axis for clarity. The vertical dotted red line indicates the diamagnetic resonance field as a reference. The green and red solid lines represent the intrinsic and impurity contributions, respectively, in the total spectra (black solid line). The green arrow marks the peak position of the intrinsic line. (b) Normalized ${ }^{115}$ In NMR spectra at different temperatures between 1.45 and $24 \mathrm{mK}$. The green, red, and black solid lines correspond to the spectra at $24 \mathrm{mK}$. (c) NMR spectra measured with different pulse separations $\tau$ at $25 \mathrm{~K}$.

temperature-dependent part of the line shift $1 /\left(K-K_{0}\right)$ is shown as a function of temperature in the inset of Fig. 7(a). A $\mathrm{CW}$ fit of the data yields the $\mathrm{CW}$ temperature $\theta_{\mathrm{NMR}}=-14 \mathrm{~K}$. This value should be more reliable than $\theta_{\chi}=-7 \mathrm{~K}$ estimated from the bulk susceptibility since the NMR line shift does not contain any impurity contributions.

At low temperature, the total spectra remain unchanged, as shown in Fig. 8(b). This indicates that both the line shift $K$ and linewidth $\Delta H$ of the spectra remain constant down to the base temperature of $25 \mathrm{mK}$. Saturation of both these quantities suggests saturation of spin correlations below $\sim 4 \mathrm{~K}$. The finite and temperature-independent value of $K$ below $4 \mathrm{~K}$ gives strong evidence for the gapless behavior. From $\Delta H$, we estimated the saturated magnetic moment $\mu_{s}=0.89 \mu_{B} /$ f.u., close to $1 \mu_{B}$ expected for one unpaired electron per $\operatorname{Ir}_{2} \mathrm{O}_{9}$ dimer. 
To probe the low-energy spin dynamics, we have studied the NMR spin-lattice relaxation rate $\left(1 / T_{1}\right)$ at various temperatures. The rate $1 / T_{1}$ was measured at the peak position of the intrinsic line using long pulses to avoid any interference from the impurity line. At different temperatures, $1 / T_{1}$ are obtained by fitting [shown in the inset of Fig. 7(b)] the longitudinal nuclear magnetization recovery curves with the equation

$$
\begin{aligned}
1-M_{t} / M_{\infty}= & C\left(0.006 e^{-(2 W t)^{\beta}}+0.0335 e^{-(12 W t)^{\beta}}\right. \\
& +0.0925 e^{-(30 W t)^{\beta}}+0.215 e^{-(56 W t)^{\beta}} \\
& \left.+0.653 e^{-(90 W t)^{\beta}}\right),
\end{aligned}
$$

where $1 / T_{1}=2 W$. Here, $M$ is the nuclear magnetization, $\beta$ is a stretched exponent, which accounts for a distribution of the $T_{1}$ values due to disorder, and $C$ is a prefactor.

In general,

$$
\left(1 / T_{1} T\right) \sim \sum_{q} A_{\mathrm{hf}}(q)^{2} \chi^{\prime \prime}(q, \omega \rightarrow 0),
$$

where $\chi^{\prime \prime}(q, \omega)$ is the imaginary part of the dynamical spin susceptibility. The temperature dependence of $1 / T_{1}$ is shown in Fig. 7(b). Between 30 and $4 \mathrm{~K}, 1 / T_{1}$ is almost temperature-independent, indicating paramagnetic behavior. A weak humplike feature is seen around $1.6 \mathrm{~K}$, which coincides with the hump in the $C_{p} / T$ data [Fig. 4(a)] and with the rapid increase in $\lambda_{\text {ZF }}$ [Fig. $6(\mathrm{~b})$ ]. No long-range order occurs in $\mathrm{Ba}_{3} \mathrm{InIr}_{2} \mathrm{O}_{9}$. Therefore, this hump is not a broadened signature of a magnetic transition. Instead, it may indicate some intrinsic physics, as recently proposed for the honeycomb iridates [49]. The applicability of this scenario to $\mathrm{Ba}_{3} \operatorname{InIr}_{2} \mathrm{O}_{9}$ may be an interesting venue for future research.

Below $0.3 \mathrm{~K},\left(1 / T_{1}\right)$ follows the $\sim T^{2.2}$ power law, similar to the $\sim T^{1.83}$ behavior of $C_{m}(T)$.

\section{DISCUSSION AND SUMMARY}

Our results establish a gapless ground state and persistent spin dynamics down to at least $20 \mathrm{mK}$, rendering $\mathrm{Ba}_{3} \mathrm{InIr}_{2} \mathrm{O}_{9}$ a potential QSL material. However, its low-temperature behavior is rather different from theoretical expectations for a gapless QSL, for which magnetic excitations are usually described in terms of a spinon Fermi surface and should give rise to the linear behavior of both the spin-lattice relaxation rate and magnetic specific heat [50-52] at low temperatures. Our data for $\mathrm{Ba}_{3} \mathrm{InIr}_{2} \mathrm{O}_{9}$ clearly deviate from this scenario.

Microscopically, we expect two superexchange pathways. The mixed-valence $\operatorname{Ir}_{2} \mathrm{O}_{9}$ dimers may interact in the $a b$ plane via the coupling $J_{T}$ (an Ir-Ir distance of $5.83 \AA$ ) and along the $c$ direction via the coupling $J_{H}$ (an Ir-Ir distance of $5.71 \AA$; see Fig. 1). Note that we consider Ir-Ir distances because magnetic electrons are expected to occupy a molecular orbital of the dimer with equal contributions of both Ir sites [17]. While the coupling $J_{T}$ forms a triangular lattice in the $a b$ plane, as already anticipated in $\mathrm{Ba}_{3} \mathrm{IrTi}_{2} \mathrm{O}_{9}$ [21-25], the coupling $J_{H}$ leads to a buckled honeycomb geometry. Depending on the ratio between $J_{T}$ and $J_{H}$, the system can interpolate between the purely two-dimensional regime $\left(\left|J_{T}\right| \gg\left|J_{H}\right|\right.$ or vice versa) and a three-dimensional behavior when both $\left|J_{H}\right|$ and $\left|J_{T}\right|$ are of the same size.
On the phenomenological level, the quadratic behavior of the zero-field specific heat is expected in an algebraic spin liquid on the kagome lattice [53,54]. Another manifestation of the $T^{2}$ behavior was proposed for a spin model combining the triangular and honeycomb geometries [55], as in $\mathrm{Ba}_{3} \mathrm{InIr}_{2} \mathrm{O}_{9}$, but its applicability to our system is obscured by the large single-ion anisotropy term that is central to the model but seems unlikely in iridates [15]. Intriguingly, the robust quadratic behavior of the zero-field specific heat has been reported in frustrated magnets with slow dynamics $[56,57]$ and interpreted in the framework of Halperin-Saslow modes for two-dimensional spin glasses [58,59]. It was also observed in $\mathrm{Li}_{2} \mathrm{RhO}_{3}$, the Kitaev antiferromagnet, in which spins freeze below $6 \mathrm{~K}$ [60]. To what extent these scenarios apply to $\mathrm{Ba}_{3} \mathrm{InIr}_{2} \mathrm{O}_{9}$ with its clear signatures of persistent spin dynamics should be the subject of future investigations.

Mixed-valence dimers are also reported in ruthenates with the hexagonal perovskite structure. However, these compounds show long-range magnetic order or disordered static magnetism at low temperatures [37]. This is different from our case, where persistent spin dynamics is observed. From a magnetism point of view, mixed-valence hexagonal perovskites remain a largely uncharted territory. The nature of local moments within the mixed-valence dimers requires further investigation, and peculiarities of magnetic interactions between the dimers remain to be explored. Experiments reveal instances of nontrivial magnetic states, including the potential QSL state in $\mathrm{Ba}_{3} \mathrm{InIr}_{2} \mathrm{O}_{9}$, and call for a better understanding from theoretical and microscopic perspectives.

Altogether, we demonstrated that $\mathrm{Ba}_{3} \mathrm{InIr}_{2} \mathrm{O}_{9}$ is a structurally well ordered QSL candidate showing the $\sim T^{1.83}$ behavior of zero-field specific heat and the $\sim T^{2.2}$ power law for the NMR spin-lattice relaxation rate at low temperatures. Not only would it be interesting for further experimental (a probe of the spin excitations) and theoretical (microscopic analysis of the magnetic model) research, it also puts forward mixed-valence iridates as a promising playground for finding new QSL materials. The unique combination of the triangular and buckled honeycomb geometries bears strong connections to current theoretical models of frustrated magnetism [13,21,61-64] and may lead to novel manifestations of the QSL physics when different lattice geometries agree.

\section{ACKNOWLEDGMENTS}

We gratefully acknowledge financial support from the Deutsche Forschungsgemeinschaft (DFG) within the collaborative research center TRR 80 (Augsburg/Munich). T.D., M.M., and A.A.T. were funded by the Federal Ministry for Education and Research through the Sofja Kovalevskaya Award of the Alexander von Humboldt Foundation. F.B. acknowledges financial support from the project SOCRATE (ANR-15-CE30-0009-01) of the French agency ANR. P.K. acknowledges support from the European Commission through Marie Curie International Incoming Fellowship (PIIF-GA2013-627322). Part of the work was performed at the LTF and DOLLY spectrometers of the Swiss Muon Source (Paul Scherrer Institute, Villigen, Switzerland). We thank C. Baines for his support on LTF and D. Vieweg for her help with high-temperature susceptibility measurements. 
[1] L. Balents, Spin liquids in frustrated magnets, Nature (London) 464, 199 (2010).

[2] L. Savary and L. Balents, Quantum spin liquids: A review, Rep. Prog. Phys. 80, 016502 (2017).

[3] P. W. Anderson, Resonating valence bonds: A new kind of insulator? Mater. Res. Bull. 8, 153 (1973).

[4] S. Yan, D. A. Huse, and S. R. White, Spin-liquid ground state of the $S=1 / 2$ kagome Heisenberg antiferromagnet, Science 332, 1173 (2011).

[5] R. V. Mishmash, J. R. Garrison, S. Bieri, and C. Xu, Theory of a Competitive Spin Liquid State for Weak Mott Insulators on the Triangular Lattice, Phys. Rev. Lett. 111, 157203 (2013).

[6] R. Kaneko, S. Morita, and M. Imada, Gapless spin-liquid phase in an extended spin-1/2 triangular Heisenberg model, J. Phys. Soc. Jpn. 83, 093707 (2014).

[7] W.-J. Hu, S.-S. Gong, W. Zhu, and D. N. Sheng, Competing spin-liquid states in the spin- $\frac{1}{2}$ Heisenberg model on the triangular lattice, Phys. Rev. B 92, 140403(R) (2015).

[8] Y. Iqbal, W.-J. Hu, R. Thomale, D. Poilblanc, and F. Becca, Spin liquid nature in the Heisenberg $J_{1}-J_{2}$ triangular antiferromagnet, Phys. Rev. B 93, 144411 (2016).

[9] M. R. Norman, Colloquium: Herbertsmithite and the search for the quantum spin liquid, Rev. Mod. Phys. 88, 041002 (2016).

[10] B. Fåk, E. Kermarrec, L. Messio, B. Bernu, C. Lhuillier, F. Bert, P. Mendels, B. Koteswararao, F. Bouquet, J. Ollivier, A. D. Hillier, A. Amato, R. H. Colman, and A. S. Wills, Kapellasite: A Kagome Quantum Spin Liquid with Competing Interactions, Phys. Rev. Lett. 109, 037208 (2012).

[11] L. Clark, J. C. Orain, F. Bert, M. A. De Vries, F. H. Aidoudi, R. E. Morris, P. Lightfoot, J. S. Lord, M. T. F. Telling, P. Bonville, J. P. Attfield, P. Mendels, and A. Harrison, Gapless Spin Liquid Ground State in the $S=\frac{1}{2}$ Vanadium Oxyfluoride Kagome Antiferromagnet $\left[\mathrm{NH}_{4}\right]_{2}\left[\mathrm{C}_{7} \mathrm{H}_{14} \mathrm{~N}\right]\left[\mathrm{V}_{7} \mathrm{O}_{6} \mathrm{~F}_{18}\right]$, Phys. Rev. Lett. 110, 207208 (2013).

[12] C. Balz, B. Lake, J. Reuther, H. Luetkens, R. Schönemann, T. Herrmannsdörfer, Y. Singh, A. T. M. Nazmul Islam, E. M. Wheeler, J. A. Rodriguez-Rivera, T. Guidi, G. G. Simeoni, C. Baines, and H. Ryll, Physical realization of a quantum spin liquid based on a complex frustration mechanism, Nat. Phys. 12, 942 (2016).

[13] A. Kitaev, Anyons in an exactly solved model and beyond, Ann. Phys. (NY) 321, 2 (2006).

[14] G. Jackeli and G. Khaliullin, Mott Insulators in the Strong SpinOrbit Coupling Limit: From Heisenberg to a Quantum Compass and Kitaev Models, Phys. Rev. Lett. 102, 017205 (2009).

[15] S. M. Winter, Y. Li, H. O. Jeschke, and R. Valentí, Challenges in design of Kitaev materials: Magnetic interactions from competing energy scales, Phys. Rev. B 93, 214431 (2016).

[16] S. M. Winter, A. A. Tsirlin, M. Daghofer, J. van den Brink, Y. Singh, P. Gegenwart, and R. Valentí, Models and materials for generalized Kitaev magnetism, arXiv:1706.06113 [J. Phys.: Condens. Matter (to be published)].

[17] S. V. Streltsov and D. I. Khomskii, Covalent bonds against magnetism in transition metal compounds, Proc. Natl. Acad. Sci. USA 113, 10491 (2016).

[18] Y. Shirata, H. Tanaka, A. Matsuo, and K. Kindo, Experimental Realization of a Spin-1/2 Triangular-Lattice Heisenberg Antiferromagnet, Phys. Rev. Lett. 108, 057205 (2012).

[19] H. D. Zhou, C. Xu, A. M. Hallas, H. J. Silverstein, C. R. Wiebe, I. Umegaki, J. Q. Yan, T. P. Murphy, J.-H. Park, Y. Qiu,
J. R. D. Copley, J. S. Gardner, and Y. Takano, Successive Phase Transitions and Extended Spin-Excitation Continuum in the $S=\frac{1}{2}$ Triangular-Lattice Antiferromagnet $\mathrm{Ba}_{3} \mathrm{CoSb}_{2} \mathrm{O}_{9}$, Phys. Rev. Lett. 109, 267206 (2012).

[20] J. Ma, Y. Kamiya, T. Hong, H. B. Cao, G. Ehlers, W. Tian, C. D. Batista, Z. L. Dun, H. D. Zhou, and M. Matsuda, Static and Dynamical Properties of the Spin-1/2 Equilateral Triangular-Lattice Antiferromagnet $\mathrm{Ba}_{3} \mathrm{CoSb}_{2} \mathrm{O}_{9}$, Phys. Rev. Lett. 116, 087201 (2016).

[21] M. Becker, M. Hermanns, B. Bauer, M. Garst, and S. Trebst, Spin-orbit physics of $j=\frac{1}{2}$ Mott insulators on the triangular lattice, Phys. Rev. B 91, 155135 (2015).

[22] A. Catuneanu, J. G. Rau, H.-S. Kim, and H.-Y. Kee, Magnetic orders proximal to the Kitaev limit in frustrated triangular systems: Application to $\mathrm{Ba}_{3} \mathrm{IrTi}_{2} \mathrm{O}_{9}$, Phys. Rev. B 92, 165108 (2015).

[23] T. Dey, A. V. Mahajan, P. Khuntia, M. Baenitz, B. Koteswararao, and F. C. Chou, Spin-liquid behavior in $J_{\text {eff }}=\frac{1}{2}$ triangular lattice compound $\mathrm{Ba}_{3} \operatorname{IrTi}_{2} \mathrm{O}_{9}$, Phys. Rev. B 86, 140405(R) (2012).

[24] R. Kumar, D. Sheptyakov, P. Khuntia, K. Rolfs, P. G. Freeman, H. M. Rønnow, T. Dey, M. Baenitz, and A. V. Mahajan, $\mathrm{Ba}_{3} M_{x} \mathrm{Ti}_{3-x} \mathrm{O}_{9}(M=\mathrm{Ir}, \mathrm{Rh})$ : A family of $5 d / 4 d$-based diluted quantum spin liquids, Phys. Rev. B 94, 174410 (2016).

[25] W.-J. Lee, S.-H. Do, S. Yoon, S. Lee, Y. S. Choi, D. J. Jang, M. Brando, M. Lee, E. S. Choi, S. Ji, Z. H. Jang, B. J. Suh, and K.-Y. Choi, Putative spin liquid in the triangle-based iridate $\mathrm{Ba}_{3} \mathrm{IrTi}_{2} \mathrm{O}_{9}$, Phys. Rev. B 96, 014432 (2017).

[26] J. Darriet, M. Drillon, G. Villeneuve, and P. Hagenmuller, Interactions magnétiques dans des groupements binucléaires du Ruthénium +V, J. Solid State Chem. 19, 213 (1976).

[27] J. Darriet, J. L. Soubeyroux, and A. P. Murani, Neutron inelastic scattering study of exchange interactions in a ruthenium(V) dimer $\mathrm{Ba}_{3} \mathrm{CaRu}_{2} \mathrm{O}_{9}$, J. Phys. Chem. Solids 44, 269 (1983).

[28] Y. Doi and Y. Hinatsu, The structural and magnetic characterization of $6 \mathrm{H}$-perovskite-type oxides $\mathrm{Ba}_{3} \mathrm{LnIr}_{2} \mathrm{O}_{9}(\mathrm{Ln}=\mathrm{Y}$, lanthanides), J. Phys. Condens. Matter 16, 2849 (2004).

[29] T. Sakamoto, Y. Doi, and Y. Hinatsu, Crystal structures and magnetic properties of $6 \mathrm{H}$-perovskite-type oxides $\mathrm{Ba}_{3} \mathrm{MIr}_{2} \mathrm{O}_{9}$ $(\mathrm{M}=\mathrm{Mg}, \mathrm{Ca}, \mathrm{Sc}, \mathrm{Ti}, \mathrm{Zn}, \mathrm{Sr}, \mathrm{Zr}, \mathrm{Cd}$ and In), J. Solid State Chem. 179, 2595 (2006).

[30] T. Dey, A. V. Mahajan, R. Kumar, B. Koteswararao, F. C. Chou, A. A. Omrani, and H. M. Ronnow, Possible spin-orbit driven spin-liquid ground state in the double perovskite phase of $\mathrm{Ba}_{3} \mathrm{YIr}_{2} \mathrm{O}_{9}$, Phys. Rev. B 88, 134425 (2013).

[31] T. Dey, R. Kumar, A. V. Mahajan, S. D. Kaushik, and V. Siruguri, Unconventional magnetism in the spin-orbit-driven Mott insulators $\mathrm{Ba}_{3} M \mathrm{Ir}_{2} \mathrm{O}_{9}(M=\mathrm{Sc}, \mathrm{Y})$, Phys. Rev. B 89, 205101 (2014).

[32] M. Hoelzel, A. Senyshyn, N. Juenke, H. Boysen, W. Schmahl, and $\mathrm{H}$. Fuess, High-resolution neutron powder diffractometer SPODI at research reactor FRM II, Nucl. Instrum. Methods Phys. Res., Sect. A 667, 32 (2012).

[33] V. Petř́íček, M. Dušek, and L. Palatinus, Crystallographic computing system JANA2006: General features, Z. Kristallogr. Cryst. Mater. 229, 345 (2014).

[34] C. D. Ling, B. J. Kennedy, Q. Zhou, J. R. Spencer, and M. Avdeev, Synthesis, structures, and phase transitions of barium bismuth iridium oxide perovskites $\mathrm{Ba}_{2} \mathrm{BiIrO}_{6}$ and $\mathrm{Ba}_{3} \mathrm{BiIr}_{2} \mathrm{O}_{9}$, J. Solid State Chem. 183, 727 (2010). 
[35] J. Terzic, J. C. Wang, F. Ye, W. H. Song, S. J. Yuan, S. Aswartham, L. E. DeLong, S. V. Streltsov, D. I. Khomskii, and G. Cao, Coexisting charge and magnetic orders in the dimer-chain iridate $\mathrm{Ba}_{5} \mathrm{AlIr}_{2} \mathrm{O}_{11}$, Phys. Rev. B 91, 235147 (2015).

[36] We introduced the site mixing and constrained the overall composition to $\mathrm{Ba}_{3} \mathrm{InIr}_{2} \mathrm{O}_{9}$. This leads to $R_{I}=0.038$ and $R_{p}=0.050$ compared to $R_{I}=0.039$ and $R_{p}=0.051$ for the fully ordered model (room-temperature data).

[37] D. Ziat, A. A. Aczel, R. Sinclair, Q. Chen, H. D. Zhou, T. J. Williams, M. B. Stone, A. Verrier, and J. A. Quilliam, Frustrated spin- $\frac{1}{2}$ molecular magnetism in the mixed-valence antiferromagnets $\mathrm{Ba}_{3} M \mathrm{Ru}_{2} \mathrm{O}_{9}(M=\mathrm{In}, \mathrm{Y}, \mathrm{Lu})$, Phys. Rev. B 95, 184424 (2017).

[38] W. Miiller, M. Avdeev, Q. Zhou, B. J. Kennedy, N. Sharma, R. Kutteh, G. J. Kearley, S. Schmid, K. S. Knight, P. E. R. Blanchard, and C. D. Ling, Giant magnetoelastic effect at the opening of a spin-gap in $\mathrm{Ba}_{3} \mathrm{BiIr}_{2} \mathrm{O}_{9}$, J. Am. Chem. Soc. 134, 3265 (2012).

[39] Y. Li, H. Liao, Z. Zhang, S. Li, F. Jin, L. Ling, L. Zhang, Y. Zou, L. Pi, Z. Yang, J. Wang, Z. Wu, and Q. Zhang, Gapless quantum spin liquid ground state in the two-dimensional spin$1 / 2$ triangular antiferromagnet $\mathrm{YbMgGaO}_{4}$, Sci. Rep. 5, 16419 (2015).

[40] Y. Li, D. Adroja, P. K. Biswas, P. J. Baker, Q. Zhang, J. Liu, A. A. Tsirlin, P. Gegenwart, and Q. Zhang, Muon Spin Relaxation Evidence for the U(1) Quantum Spin-Liquid Ground State in the Triangular Antiferromagnet $\mathrm{YbMgGaO}_{4}$, Phys. Rev. Lett. 117, 097201 (2016).

[41] See Supplemental Material at http://link.aps.org/supplemental/ 10.1103/PhysRevB.96.174411 for details of synthesis, structure refinement, thermodynamic measurements, NMR, and $\mu \mathrm{SR}$ data.

[42] P. Sindzingre, G. Misguich, C. Lhuillier, B. Bernu, L. Pierre, Ch. Waldtmann, and H.-U. Everts, Magnetothermodynamics of the Spin-1/2 Kagomé Antiferromagnet, Phys. Rev. Lett. 84, 2953 (2000).

[43] J. Nasu, M. Udagawa, and Y. Motome, Thermal fractionalization of quantum spins in a Kitaev model: Temperature-linear specific heat and coherent transport of Majorana fermions, Phys. Rev. B 92, 115122 (2015).

[44] N. Bloembergen, E. M. Purcell, and R. V. Pound, Relaxation effects in nuclear magnetic resonance absorption, Phys. Rev. 73, 679 (1948).

[45] R. H. Colman, F. Bert, D. Boldrin, A. D. Hillier, P. Manuel, P. Mendels, and A. S. Wills, Spin dynamics in the $S=1 / 2$ quantum kagome compound vesignieite, $\mathrm{Cu}_{3} \mathrm{Ba}\left(\mathrm{VO}_{5} \mathrm{H}\right)_{2}$, Phys. Rev. B 83, 180416(R) (2011).

[46] E. Kermarrec, P. Mendels, F. Bert, R. H. Colman, A. S. Wills, P. Strobel, P. Bonville, A. Hillier, and A. Amato, Spinliquid ground state in the frustrated kagome antiferromagnet $\mathrm{MgCu}_{3}(\mathrm{OH})_{6} \mathrm{Cl}_{2}$, Phys. Rev. B 84, 100401(R) (2011).

[47] M. Gomilšek, M. Klanjšek, M. Pregelj, F. C. Coomer, H. Luetkens, O. Zaharko, T. Fennell, Y. Li, Q. M. Zhang, and A. Zorko, Instabilities of spin-liquid states in a quantum kagome antiferromagnet, Phys. Rev. B 93, 060405(R) (2016).

[48] J. C. Orain, L. Clark, F. Bert, P. Mendels, P. Attfield, F. H. Aidoudi, R. E. Morris, P. Lightfoot, A. Amato, and C. Baines, $\mu$ SR study of a quantum spin liquid candidate: The $S=1 / 2$ vanadium oxyfluoride kagome antiferromagnet, J. Phys. Conf. Ser. 551, 012004 (2014).

[49] J. Yoshitake, J. Nasu, and Y. Motome, Fractional Spin Fluctuations as a Precursor of Quantum Spin Liquids: Majorana Dynamical Mean-Field Study for the Kitaev Model, Phys. Rev. Lett. 117, 157203 (2016).

[50] M. J. Lawler, A. Paramekanti, Y. B. Kim, and L. Balents, Gapless Spin Liquids on the Three-Dimensional Hyperkagome Lattice of $\mathrm{Na}_{4} \mathrm{Ir}_{3} \mathrm{O}_{8}$, Phys. Rev. Lett. 101, 197202 (2008).

[51] S. Yamashita, Y. Nakazawa, M. Oguni, Y. Oshima, H. Nojiri, Y. Shimizu, K. Miyagawa, and K. Kanoda, Thermodynamic properties of a spin-1/2 spin-liquid state in a kappa-type organic salt, Nat. Phys. 4, 459 (2008).

[52] S. Yamashita, T. Yamamoto, Y. Nakazawa, M. Tamura, and R. Kato, Gapless spin liquid of an organic triangular compound evidenced by thermodynamic measurements, Nat. Commun. 2, 275 (2011).

[53] Y. Ran, M. Hermele, P. A. Lee, and X.-G. Wen, ProjectedWave-Function Study of the Spin-1/2 Heisenberg Model on the Kagomè Lattice, Phys. Rev. Lett. 98, 117205 (2007).

[54] M. Hermele, Y. Ran, P. A. Lee, and X.-G. Wen, Properties of an algebraic spin liquid on the kagome lattice, Phys. Rev. B 77, 224413 (2008).

[55] G. Chen, M. Hermele, and L. Radzihovsky, Frustrated Quantum Critical Theory of Putative Spin-Liquid Phenomenology in $6 \mathrm{H}$ B-Ba $\mathrm{NiSb}_{2} \mathrm{O}_{9}$, Phys. Rev. Lett. 109, 016402 (2012).

[56] A. P. Ramirez, G. P. Espinosa, and A. S. Cooper, Elementary excitations in a diluted antiferromagnetic kagomé lattice, Phys. Rev. B 45, 2505 (1992).

[57] S. Nakatsuji, Y. Nambu, H. Tonomura, O. Sakai, S. Jonas, C. Broholm, H. Tsunetsugu, Y. Qiu, and Y. Maeno, Spin disorder on a triangular lattice, Science 309, 1697 (2005).

[58] S. Sachdev, Kagomé- and triangular-lattice Heisenberg antiferromagnets: Ordering from quantum fluctuations and quantumdisordered ground states with unconfined bosonic spinons, Phys. Rev. B 45, 12377 (1992).

[59] D. Podolsky and Y. B. Kim, Halperin-Saslow modes as the origin of the low-temperature anomaly in $\mathrm{NiGa}_{2} \mathrm{~S}_{4}$, Phys. Rev. B 79, 140402(R) (2009).

[60] P. Khuntia, S. Manni, F. R. Foronda, T. Lancaster, S. J. Blundell, P. Gegenwart, and M. Baenitz, Local magnetism and spin dynamics of the frustrated honeycomb rhodate $\mathrm{Li}_{2} \mathrm{RhO}_{3}$, Phys. Rev. B 96, 094432 (2017).

[61] I. Rousochatzakis, J. Reuther, R. Thomale, S. Rachel, and N. B. Perkins, Phase Diagram and Quantum Order by Disorder in the Kitaev $K_{1}-K_{2}$ Honeycomb Magnet, Phys. Rev. X 5, 041035 (2015).

[62] G. Jackeli and A. Avella, Quantum order by disorder in the Kitaev model on a triangular lattice, Phys. Rev. B 92, 184416 (2015).

[63] K. Li, S.-L. Yu, and J.-X. Li, Global phase diagram, possible chiral spin liquid, and topological superconductivity in the triangular Kitaev-Heisenberg model, New J. Phys. 17, 043032 (2015).

[64] I. Rousochatzakis, U. K. Rössler, J. van den Brink, and M. Daghofer, Kitaev anisotropy induces mesoscopic $\mathbb{Z}_{2}$ vortex crystals in frustrated hexagonal antiferromagnets, Phys. Rev. B 93, 104417 (2016). 